\title{
ON ANALYTIC SOLUTIONS OF DIFFERENTIAL EQUATIONS IN THE NEIGHBORHOOD OF NON-ANALYTIC SINGULAR POINTS*
}

\author{
BY B. O. KOOPMAN $\dagger$
}

We shall consider the system

$$
\frac{d x_{1}}{X_{1}}=\frac{d x_{2}}{X_{2}}=\cdots=\frac{d x_{n}}{X_{n}}
$$

in the complex neighborhood of a point, say $(0,0, \cdots, 0)$, at which the $X$ 's vanish simultaneously. We shall suppose throughout that

$$
\begin{array}{r}
X_{i}=a_{i 1} x_{1}+a_{i 2} x_{2}+\cdots+a_{i n} x_{n}+\phi_{i}\left(x_{1}, \cdots, x_{n}\right), \\
(i=1,2, \cdots, n),
\end{array}
$$

and that the general solutions of the related linear system

$$
\frac{d x_{1}}{a_{11} x_{1}+\cdots+a_{1 n} x_{n}}=\cdots=\frac{d x_{n}}{a_{n 1} x_{1}+\cdots+a_{n n} x_{n}}
$$

approach zero, without being identically zero. $\ddagger$ Consider a solution of the equations (2). When will there exist an analytic solution of the equations (1) which behaves essentially like this near $(0,0, \cdots, 0)$ ? How many such solutions will there be? Our object is to answer these questions without restricting the functions $\phi_{i}$, except as regards their behavior in a certain region of the complex $x_{1} x_{2} \cdots x_{n}$-space abutting on the origin, in which the given solution of (2) is embedded. We shall thus obtain a treatment less restricted than those of Briot and Bouquet, Poincaré, Picard, Dulac, Horn, etc., who assume the $\phi$ 's to be convergent power series of terms of degree higher than the first. $\S$

* Presented to the Society, October 30, 1926.

$\dagger$ National Research Fellow in Mathematics.

$\ddagger$ See, however, the concluding note of this paper.

$\S$ For full references, see J. Malmquist, Sur les points singuliers des équations différentielles, Arkiv för Matematik, Astronomi och Fysik, vol. 15, No. 3, (1920), pp. 1-5. 
It is necessary first of all to reduce the system to a standard form. Let $\lambda_{1}, \lambda_{2}, \cdots, \lambda_{n}$ be the roots of the equation

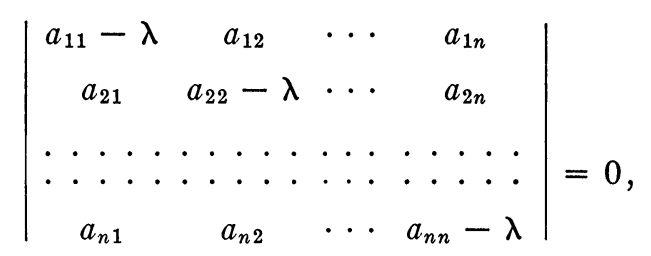

and $r_{1}, r_{2} \cdots, r_{\nu}$ their respective multiplicities $\left(r_{1}+\cdots\right.$ $\left.+r_{\nu}=n\right)$. Then we may, according to the classical theory, ${ }^{*}$ transform the equations (1) by means of a linear substitution of the $x$ 's so that the expressions for the new $X$ 's in terms of the new $x$ 's become

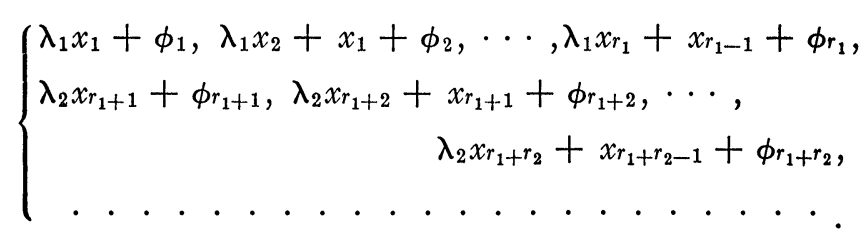

It is understood here, as in all cases where a transformation of the $x$ 's is made, that the new $\phi$ 's denote that part of the new $X$ 's which contains the old $\phi$ 's. Now the general solutions of the new equations (2) corresponding to the new values (3) of the $X$ 's are of the form $\dagger$

$$
\left\{\begin{aligned}
& x_{i}=\left(C_{1} \frac{t^{i-1}}{(i-1) !}+C_{2} \frac{t^{i-2}}{(i-2) !}+\cdots\right. \\
&\left.+C_{i-1} t+C_{i}\right) e^{\lambda_{1} t}, \quad\left(i=1,2, \cdots, r_{1}\right), \\
& x_{r_{1}+i}=\left(C_{1}^{\prime} \frac{t^{i-1}}{(i-1) !}+C_{2}^{\prime} \frac{t^{i-2}}{(i-2) !}+\cdots\right. \\
&\left.+C_{i-1}^{\prime} t+C_{i}^{\prime}\right) e^{\lambda_{2} t},\left(i=1,2, \cdots, r_{2}\right), \\
& . \quad . \quad . \quad . \quad . \quad . \quad . . .
\end{aligned}\right.
$$

* See e. g., E. Goursat, Cours d'A nalyse Mathématique, Paris, GauthierVillars, 1918, vol. II, p. 495.

$\dagger$ See Goursat, loc. cit. 
Here, $d t$ is the common ratio in (2). With the aid of these expressions it is easy to prove that, in the case we are considering, where the general solution of (2) approaches $(0,0, \cdots, 0)$, the points $\lambda_{i}$ all lie on the side away from the origin of a straight line, $l: \arg \lambda=c$, drawn in the $\lambda$-plane, not through the origin. Now the transformation of the $x$ 's which leads to the expressions (3) may be so conducted that $\lambda_{1}^{\prime}=1$, while the real parts of $\lambda_{2}, \cdots, \lambda_{\nu}$ are not less than 1 . This is accomplished by dividing the $X$ 's through by the appropriate $\lambda^{\prime} e^{c-\pi / 2}$, say $\lambda_{1}^{\prime} e^{c-\pi / 2}$, at a certain stage of the transformation. Furthermore, the effect of performing the substitution

$$
x_{r_{1}+1}=x_{1} x_{r_{1}+1}^{\prime}, \quad x_{r_{1}+2}=x_{1} x_{r_{1}+2}^{\prime}, \cdots, \quad x_{r_{1}+r_{2}}=x_{1} x_{r_{1}+r_{2}}^{\prime},
$$

is to replace the values in (3) by similar expressions in which $\lambda_{2}$ is replaced by $\lambda_{2}-\lambda_{1}$. Thus we may assume that the real parts of $\lambda_{2}, \cdots, \lambda_{\nu}$ are all less than 2 .

Consider then the system

$$
\frac{d x_{i}}{d t}=X_{i}, \quad(i=1,2, \cdots, n)
$$

where the $X$ 's have the values (3), and where

$1 \leqq \lambda_{i}^{\prime}<2, \quad\left(\lambda_{i}=\lambda_{i}^{\prime}+(-1)^{1 / 2} \lambda_{i}^{\prime \prime}\right), \quad(i=1,2,3, \cdots, \nu)$.

Let there be given a special solution:

$$
x_{i}=f_{i}(t), \quad(i=1,2, \cdots, n)
$$

of the corresponding linear equations $(2)$; i. e., let known values of the constants $C$ in equations (4) be given. Let $T$ be the semi-infinite band in the $t$-plane defined by the inequalities $T:-\infty<t^{\prime} \leqq 0,-h<t^{\prime \prime}<h$, where $t=t^{\prime}+(-1)^{1 / 2} t^{\prime \prime}$, and $h$ is some positive constant. By means of the equations $\left(4^{\prime}\right)$ the strip $T$ goes over into a two-dimensional figure in the complex $2 n$-dimensional $x_{1} x_{2} \cdots x_{n}$-space; as the point $t$ moves out to infinity in $T$, the corresponding point $\left(x_{1}, x_{2}, \cdots, x_{n}\right)$ approaches $(0,0, \cdots, 0)$. Now consider the $2 n$-dimensional region $R$ of the complex $x_{1} x_{2} \cdots x_{n}$ - 
space corresponding to the given positive constants $\rho$, $\delta\left(<2-\lambda_{i}^{\prime}\right):$

$$
R:\left|x_{i}\right|<\rho ; \quad\left|x_{i}-f_{i}(t)\right|<e^{(2-\delta) t^{\prime}} ; \quad(i=1,2, \cdots, n) .
$$

Here $t$ is supposed to remain in $T$. Our hypotheses with regard to the functions $\phi_{i}$ are as follows:

(a) They are single-valued and analytic in the region $R$.

(b) All their first partial derivatives approach zero as $\left(x_{1}, x_{2}, \cdots, x_{n}\right)$ approaches $(0,0, \cdots, 0)$ through points of $R$.

(c) The $\phi$ 's approach zero as the square of the distance of the point $\left(x_{1}, x_{2}, \cdots, x_{n}\right)$ from $(0,0, \cdots, 0)$, regarded as points in 2n-dimensional space. Analytically, this means that there exists a constant $K$ such that

$$
\begin{array}{r}
\left|\phi_{i}\left(x_{1}, \cdots, x_{n}\right)\right|<K\left(\left|x_{1}\right|^{2}+\left|x_{2}\right|^{2}+\cdots+\left|x_{n}\right|^{2}\right), \\
(i=1,2, \cdots, n),
\end{array}
$$

for all $\left(x_{1}, x_{2}, \cdots, x_{n}\right)$ in $R$.

Under these hypotheses we shall prove that the equations ( $\left.1^{\prime}\right)$ admit the solutions $x_{i}=F_{i}(t), \quad(i=1,2, \cdots, n)$, singlevalued and analytic in a certain sub-region $T^{\prime}$ of $T$, $T^{\prime}:-\infty<t^{\prime}<\Gamma \leqq 0,-h<t^{\prime \prime}<h$, and approaching zero as $t^{\prime}=-\infty$. Furthermore, that there is a positive constant $B$ and an arbitrarily small constant $\gamma$ such that $\left|F_{i}(t)-f_{i}(t)\right|$ $<B e^{(2-\gamma) t^{\prime}},(i=1,2, \cdots, n) ;$ and that $F_{i}(t)$ is the only solution having these properties.

If the $\phi$ 's were known functions of the time, the knowledge of the solutions (4) would lead to the explicit solution of $\left(1^{\prime}\right)$ in terms of definite integrals by means of the classical methods. In the actual case, the formal process will yield integral equations which are equivalent to the equations $\left(1^{\prime}\right)$. These will be solved by the method of successive approximations.*

* The idea of extending a solution of equations like (2) to the equations (1) by the method of successive approximations has been made use of by I. Bendixon (Acta Mathematica, vol. $24(1900)$ ) and, in a form quite 
The hypothesis $(b)$ implies that the functions $\phi_{i}$ satisfy a Lipschitz condition in the region $R$ :

$$
\begin{aligned}
\mid \phi_{i}\left(x_{1}{ }^{1},\right. & \left.\cdots, x_{n}{ }^{1}\right)-\phi_{i}\left(x_{1}{ }^{2}, \cdots, x_{n}{ }^{2}\right) \mid \\
& <A\left(\left|x_{1}{ }^{1}-x_{1}{ }^{2}\right|+\cdots+\left|x_{n}{ }^{1}-x_{n}{ }^{2}\right|\right),
\end{aligned}
$$

and that the constant $A$ can be taken as small as we like provided we take $\rho$ sufficiently small. In the usual proof of (6) a "convex" region is assumed, in which the points $\left(x^{1}\right)$ and $\left(x^{2}\right)$ can always be joined by a straight line segment lying wholly in its interior.* It will be possible to apply the method to our case if we can show that the given points may always be joined by a regular curve lying in $R$, the ratio of the length of which to the distance between the points being less than a fixed constant independent of $\left(x^{1}\right)$ and $\left(x^{2}\right)$. A little computation, with the aid of the expressions (4), will show that, if $\rho$ be sufficiently small, the curve defined below has the requisite properties. Suppose that

$$
\begin{aligned}
\left|x_{1}{ }^{1}\right|^{2}+\left|x_{2}{ }^{1}\right|^{2}+ & \cdots+\left|x_{n}{ }^{1}\right|^{2} \\
& \leqq\left|x_{1}{ }^{2}\right|^{2}+\left|x_{2}{ }^{2}\right|^{2}+\cdots+\left|x_{n}{ }^{2}\right|^{2} ;
\end{aligned}
$$

through the point $\left(x^{1}\right)$ pass the curve obtained by setting $t^{\prime \prime}=0$ in equations (4) (this involves making a proper choice of the $\left.C^{\prime} \mathrm{s}\right)$. Let $\left(\bar{x}^{2}\right)$ be its point of intersection with the hypersphere through $\left(x^{2}\right)$ :

$$
\begin{aligned}
\left|x_{1}\right|^{2}+\left|x_{2}\right|^{2} & +\cdots+\left|x_{n}\right|^{2} \\
& =\left|x_{1}^{2}\right|^{2}+\left|x_{2}^{2}\right|^{2}+\cdots+\left|x_{n}^{2}\right|^{2} .
\end{aligned}
$$

Now the curve we want is composed of the straight line joining $\left(x^{2}\right)$ with $\left(\bar{x}^{2}\right)$, and of the arc of the preceding curve intercepted by $\left(x^{1}\right)$ and $\left(\bar{x}^{2}\right)$. The remainder of the proof

similar to our own, by O. Perron (Mathematische Zeitschrift, vol. 14, (1922)). These authors were studying the geometric nature of the integral curves at a singular point in the real $x y$-plane, and were not concerned with the complex domain.

* See E. Goursat, loc. cit., p. 380. 
consists in examining a line integral taken along this curve, and applying the hypothesis $(b) .^{*}$

We turn now to the proof of the existence theorem. We shall give the details of the proof for a system of the second order, leaving the obvious extension to the reader. The first typical case is that of the system $\dagger$

$$
\frac{d x}{d t}=x+\phi(x, y), \quad \frac{d y}{d t}=\lambda y+\psi(x, y),
$$

where $1 \leqq \lambda^{\prime}<2$, and where $\phi$ and $\psi$ verify the hypotheses $(a)$, (b), (c) with respect to the solution

$$
x=a e^{t}, \quad y=b e^{\lambda t} .
$$

The corresponding integral equations are found to be

$$
\begin{aligned}
& x=a e^{t}+\int_{-\infty}^{t} \phi(x, y) e^{t-\xi} d \xi, \\
& y=b e^{\lambda t}+\int_{-\infty}^{t} \psi(x, y) e^{\lambda(t-\xi)} d \xi,
\end{aligned}
$$

where $\xi$ is a variable of integration: $x=x(\xi), y=y(\xi)$ in the integrand. We have chosen $-\infty$ as the lower limit, since we are interested in solutions which differ from $\left(4^{\prime \prime}\right)$ by an amount which vanishes in comparison with $e^{t}$ as $t^{\prime}=-\infty$. The path of integration could be any curve running from the given value $t$ out to infinity in the strip $T$; but it is desirable to fix it definitely, and we shall take it to be the horizontal ray running from $t$ to the left indefinitely, i. e., $-\infty<\xi^{\prime} \leqq t$, $\xi^{\prime \prime}=t^{\prime \prime}$.

Let the successive approximations be defined by recurrence as follows:

* See Goursat, loc. cit., p. 380. We use the finiteness of the logarithmic spiral, and such curves.

$\dagger$ For simplicity in writing, we are taking the imaginary part of the coefficient of $x$ in (1") zero; but the proof in the general case is quite similar. 


$$
\begin{aligned}
& x_{0}=a e^{t}, x_{k+1}=a e^{t}+\int_{-\infty}^{t} \phi\left(x_{k}, y_{k}\right) e^{t-\xi} d \xi ; \\
& y_{0}=b e^{\lambda t}, \quad y_{k+1}=b e^{\lambda t}+\int_{-\infty}^{t} \psi\left(x_{k}, y_{k}\right) e^{\lambda(t-\xi)} d \xi ; \\
&(k=0,1,2,3, \cdots) .
\end{aligned}
$$

We have to show that the functions $x_{k}$ and $y_{k}$ all exist and are analytic, and approach limits which are the required solutions as $k=\infty$. Writing as usual

$$
u_{k}=x_{k}-x_{k-1}, \quad v_{k}=y_{k}-y_{k-1}, \quad(k=1,2,3, \cdots),
$$

we have

$$
\begin{aligned}
& x_{k}=x_{0}+u_{1}+u_{2}+\cdots+u_{k}, \\
& y_{k}=y_{0}+v_{1}+v_{2}+\cdots+v_{k},
\end{aligned}
$$

and we must examine the series

$$
\left\{\begin{array}{l}
u_{1}+u_{2}+u_{3}+\cdots \\
v_{1}+v_{2}+v_{3}+\cdots
\end{array}\right.
$$

for convergence.

From the expression

$$
u_{1}=\int_{-\infty}^{t} \phi\left(x_{0}, y_{0}\right) e^{t-\xi} d \xi,
$$

and the hypothesis $(c)$, we have

$$
\begin{aligned}
\left|u_{1}\right| & \leqq \int_{-\infty}^{t^{\prime}}\left|\phi\left(x_{0}, y_{0}\right)\right| e^{t^{\prime}-\xi^{\prime}} d \xi^{\prime}, \quad\left(\xi=\xi^{\prime}+(-1)^{1 / 2} \xi^{\prime \prime}\right) \\
& <K \int_{-\infty}^{t^{\prime}}\left(\left|x_{0}\right|^{2}+\left|y_{0}\right| 2\right) e^{t^{\prime}-\xi^{\prime}} d \xi^{\prime} \\
& <K e^{t^{\prime}} \int_{-\infty}^{t^{\prime}}\left(|a|^{2} e^{\xi^{\prime}}+|b|^{2} e^{\left.\left(2 \lambda^{\prime}-1\right) \xi^{\prime}-2 \lambda^{\prime \prime} t^{\prime \prime}\right) d \xi^{\prime}}\right. \\
& <K\left(|a|^{2}+\frac{|b|^{2}}{2 \lambda^{\prime}-1} e^{2\left(\lambda^{\prime}-1\right) t^{\prime}-2 \lambda^{\prime \prime} t^{\prime \prime}}\right) e^{2 t^{\prime}}
\end{aligned}
$$


Similarly,

(9) $\quad\left|v_{1}\right|<K\left(\frac{|a|^{2}}{2-\lambda^{\prime}}+\frac{|b|^{2}}{\lambda^{\prime}} e^{2\left(\lambda^{\prime}-1\right) t^{\prime}-2 \lambda^{\prime \prime} t^{\prime \prime}}\right) e^{2 t^{\prime}}$.

Hence it is possible to find a positive constant $M$ such that

$$
\left|u_{1}\right|<M e^{2 t^{\prime}}, \quad\left|v_{1}\right|<M e^{2 t^{\prime}} .
$$

Moreover, the preceding computation establishes the existence and analyticity of the functions $u_{1}, v_{1}$ in $T$, as it follows from the elementary considerations of the uniform convergence of definite integrals.

For the further $u$ 's and $v$ 's we have the equations

$$
\begin{aligned}
u_{k+1} & =\int_{-\infty}^{t}\left[\phi\left(x_{k}, y_{k}\right)-\phi\left(x_{k-1}, y_{k-1}\right)\right] e^{t-\xi} d \xi, \\
v_{k+1} & =\int_{-\infty}^{t}\left[\psi\left(x_{k}, y_{k}\right)-\psi\left(x_{k-1}, y_{k-1}\right)\right] e^{\lambda(t-\xi)} d \xi, \\
& (k=1,2,3, \cdots) .
\end{aligned}
$$

Suppose that all the functions up to and including $x_{k}, y_{k}$ exist and are analytic in the region $T$, that they map $T$ in the interior of $R$, and, finally, that there exist a positive constant $H_{k}$ for which $\left|u_{k}\right|<H_{k} e^{2 t^{\prime}},\left|v_{k}\right|<H_{k} e^{2 t^{\prime}}$. It will then follow from hypothesis $(b)$ that

$$
\begin{aligned}
\left|u_{k+1}\right| & \leqq \int_{-\infty}^{t^{\prime}}\left|\phi\left(x_{k}, y_{k}\right)-\phi\left(x_{k-1}, y_{k-1}\right)\right| e^{t^{\prime}-\xi^{\prime}} d \xi^{\prime} \\
& <A \int_{-\infty}^{t^{\prime}}\left(\left|u_{k}\right|+\left|v_{k}\right|\right) e^{t^{\prime}-\xi^{\prime}} d \xi^{\prime} \\
& <2 A H_{k} e^{t^{\prime}} \int_{-\infty}^{t^{\prime}} e^{\xi^{\prime}} d \xi^{\prime}=2 A H_{k} e^{2 t^{\prime}} . \quad[\text { See (6).] }
\end{aligned}
$$

Similarly,

$$
\left|v_{k+1}\right|<\frac{2 A H_{k}}{2-\lambda^{\prime}} e^{2 t^{\prime}}
$$


Thus, if $G$ denote the greater of the two quantities 2 and $2 /\left(2-\lambda^{\prime}\right)$, we have

$$
\left|u_{k+1}\right|<A G \cdot H_{k} e^{2 t^{\prime}}, \quad\left|v_{k+1}\right|<A G \cdot H_{k} e^{2 t^{\prime}} .
$$

Moreover, $u_{k+1}$ and $v_{k+1}$ exist and are analytic in $T$, and likewise for $x_{k+1}$ and $y_{k+1}$.

We wish to know when the locus of the corresponding point, $\left(x_{k+1}, y_{k+1}\right)$, will lie inside $R$. We have

$$
\begin{aligned}
\left|x_{k+1}-a e^{t}\right| \leqq\left|u_{1}\right|+\left|u_{2}\right|+\cdots+\left|u_{k+1}\right| \\
<\left(1+A G+A^{2} G^{2}+\cdots+A^{k} G^{k}\right) M e^{2 t^{\prime}}
\end{aligned}
$$

the last inequality resulting from the repeated application of the results of the two preceding paragraphs. Now consider the geometric series $1+A G+A^{2} G^{2}+A^{3} G^{3}+\cdots$. We shall suppose that $\rho$ is taken so small that $A G<1$; this is permissible in view of what has been deduced from hypothesis $(b)$; and in order to be sure that the loci of $\left(x_{0}, y_{0}\right), \cdots,\left(x_{k}, y_{k}\right)$ lie in this region, it is only necessary to confine $t$ to a suitable region $T^{\prime}: t^{\prime}<\Gamma$. Then the geometric series will converge, and have the sum $1 /(1-A G)$. Hence

$$
\left|x_{k+1}-a e^{t}\right|<\frac{M}{1-A G} e^{2 t^{\prime}}, \quad\left|y_{k+1}-b e^{\lambda t}\right|<\frac{M}{1-A G} e^{2 t^{\prime}} .
$$

A comparison of these inequalities with those defining $R$ shows that if $\Gamma$ in the definition of $T^{\prime}$ be given a sufficiently small value (which will be independent of $k$ ), and if $t$ be confined to $T^{\prime}$, the locus of $\left(x_{k+1}, y_{k+1}\right)$ will lie wholly within $R$.

Thus, our points $\left(x_{i}, y_{i}\right)$ will never leave $R$ as long as $t$ is in $T^{\prime}$, our terms will all be determined by recurrence, and the above inequalities will apply to them. They will be singlevalued and analytic for $t$ in $T^{\prime}$, and the series will be dominated by the convergent geometric series. It follows by the usual reasoning that $\left(x_{i}, y_{i}\right)$ converges to $(x, y)$, the required solutions of (7) and hence of $\left(1^{\prime \prime}\right)$.

The inequalities (11) evidently yield the result

$$
\left|x-a e^{t}\right|<B e^{2 t^{\prime}}, \quad\left|y-b e^{\lambda t}\right|<B e^{2 t^{\prime}} .
$$


Now there can not be more than one solution which is thus related to $\left(a e^{t}, b e^{\lambda t}\right)$. For if $\bar{x}, \bar{y}$ were a second solution, it would satisfy (7), and it would follow by previous formulas that

$$
\bar{x}-x_{k+1}=\int_{-\infty}^{t}\left[\phi(\bar{x}, \bar{y})-\phi\left(x_{k}, y_{k}\right)\right] e^{t-\xi} d \xi, \text { etc. }
$$

Hence

$$
\left|\bar{x}-x_{k+1}\right|<A \int_{-\infty}^{t^{\prime}}\left(\left|\bar{x}-x_{k}\right|+\left|\bar{y}-y_{k}\right|\right) e^{t^{\prime}-\xi^{\prime}} d \xi^{\prime}, \text { etc. }
$$

It follows by recurrence, as before, that

$$
\left|\bar{x}-x_{k}\right|<A^{k} G^{k} \cdot B e^{2 t^{\prime}}, \text { etc. ; }
$$

hence we have*

$$
x \equiv \bar{x}, \quad y \equiv \bar{y}, \quad \text { (q.e.d.). }
$$

The form of the uniqueness theorem originally stated is proved in the same manner.

We turn n'sw to the second typical case for the system of the second order. It is that of the equations

$$
\frac{d x}{d t}=x+\phi(x, y), \quad \frac{d y}{d t}=x+y+\psi(x, y) .
$$

The solution of the corresponding linear system is

$$
x=a e^{t}, \quad y=(a t+b) e^{t},
$$

and the equivalent integral equations are

$$
\left\{\begin{array}{l}
x=a e^{t}+\int_{-\infty}^{t} \phi(x, y) e^{t-\xi} d \xi \\
y=(a+b t) e^{t}+\int_{-\infty}^{t}[\psi(x, y)+(t-\xi) \phi(x, y)] e^{t-\xi} d \xi .
\end{array}\right.
$$

The solution by successive approximations is just the same as before, and the computation is performed on parallel lines. The inequality (9) is replaced by the following:

* See Goursat, loc. cit., p. 377. 


$$
\begin{aligned}
\left|v_{1}\right| & \leqq e^{t^{\prime}} \int_{-\infty}^{t^{\prime}}\left(\left|\psi_{0}\right|+|t-\xi| \cdot\left|\phi_{0}\right|\right) e^{-\xi^{\prime}} d \xi^{\prime} \\
& <K e^{t^{\prime}} \int_{-\infty}^{t^{\prime}}\left(\left|x_{0}\right|^{2}+\left|y_{0}\right|^{2}\right)(1+|t-\xi|) e^{-\xi^{\prime}} d \xi^{\prime} \\
& <K e^{t^{\prime}} \int_{-\infty}^{t^{\prime}}\left(|a|^{2}+|a \xi+b|^{2}\right)(1+|t-\xi|) e^{\xi^{\prime}} d \xi^{\prime} .
\end{aligned}
$$

Hence, if we take $t$ in a sufficiently restricted region $T^{\prime}$, we have

$$
\left|v_{1}\right|<M^{\prime} e^{t^{\prime}} \int_{-\infty}^{t^{\prime}} e^{\xi^{\prime}-\gamma} d \xi^{\prime}=M e^{(2-\gamma) t},
$$

where $\gamma$ is a positive constant which we may take as small as we wish, provided $\Gamma$ be taken sufficiently small. Likewise, to prove the inequality corresponding with (10) we have

$$
\left|u_{k}\right|<H_{k} e^{(2-\gamma) t^{\prime}}, \quad\left|v_{k}\right|<H_{k} e^{(2-\gamma) t^{\prime}},
$$

whence

$$
\begin{aligned}
\left|v_{k+1}\right| & <e^{t^{\prime}} \int_{-\infty}^{t^{\prime}} A\left(\left|u_{k}\right|+\left|v_{k}\right|\right)(1+|t-\xi|) e^{-\xi^{\prime}} d \xi^{\prime} \\
& <A H_{k} e^{t^{\prime}} \int_{-\infty}^{t^{\prime}}(1+|t-\xi|) e^{(1-\gamma) \xi^{\prime}} d \xi^{\prime}
\end{aligned}
$$

Here again, if $t$ is taken in a region $T^{\prime}$, with $\Gamma$ sufficiently small, we shall have

$$
\left|v_{k+1}\right|<H_{k} \cdot A G e^{(2-\gamma) t^{\prime}} .
$$

We leave it to the reader to complete the proof.

Note: Although we have supposed throughout that the general solution of the equations (2) approaches zero as $t=\infty$, it will be seen that even in the contrary case, if a particular solution of (2) approaches zero, it is possible to frame a theorem, like the one which has been given, which establishes the extension of the particular solution to the given differential equations (1).

Princeton University 